the lower and upper epidermis of the leaves. Probably in about ten days to two weeks the larvae complete their work as miners and begin constructing oval cases which they cut out of their mines in the leaves.

The larvae live as case-bearers during July, August, and September falling with the leaves and changing to pupae within their cases. Here among the fallen foliage the pupae remain until the following spring. There is thus but one generation each year.

\title{
Methods of Control
}

Trees standing in a pasture where the leaves are blown away even though they were only a few rods from a badly infested woodland were scarcely touched by the insect. This suggests that if the leaves were raked in piles and burned or otherwise destroyed the pest could be held in check. This, of course, would entail considerable time and labor but if well done for once only the pest would be effectively checked for several years.

The only other feasible control consists of a thorough dusting of a grove with arsenate of lead by means of an airplane which now seems to be within reasonable possibility.

\section{References}

1856. Fitch, Asa. Second Rept. Nox. Ben. Ins. N. Y., p. 269-273.

1885. Fletcher, James. Rept. of the Ent., Dept. Agri. Can., p. 14.

1903. Comstock, AnNA B. Ways of the Six-footed. Ginn \& Co., p. 29.

1912. Felr, E. P. 27th. Rept. State Ent. N. Y., p. 56-59.

\section{ARTIFICIAL PRODUCTION OF HOPPERBURN}

\section{By F. A. Fenton and I. L. Ressler \\ Iowa State College, Ames, Iowa}

While there have been numerous tests made which have shown that Empoasca mali LeB is capable of producing hopperburn when placed on potato leaves, yet there has been comparatively little done in the way of inoculation tests with this insect. The writers conducted experiments during the past summer in order to observe the effects of the injection of leafhopper extract and of dilute poisons into the leaf tissue as well as to test out further the results of multilation of potato leaves, and were able to secure interesting data which may help toward the ultimate solution of this problem. Potato leaves were mutilated with various instruments, inoculated with dilute acid and alkali, and with water in which mali adults, nymphs or hopperburned tissue had been macerated. Also further tests were made in which these insects 
were crushed on the leaf tissue or leaf petioles and in which the macerated leafhopper juice was drawn into the leaves by leaf transpiration.

\section{What IS HOPPERBURN}

There are several types of injury found on potato leaves which are characterized by the death and turring brown of the leaf tissue. These have often been confused and described under the general term tipburn. The writers believe that much of the skepticism concerning the recent work on hopperburn is because of a failure to distinguish between these various leaf disorders. It therefore seems advisable at this point to describe briefly the injury which should be characterized as hopperburn and to compare it with other related leaf burns. Hopperburn always begins at the margin of the leafand the burning is correlated with the veins. This relationship is not evident in older hopperburned leaves but is easily seen in the earlier stages (Fig. 15, 1-4) when the triangular or diamond shaped areas are seen to run in from the margin and to follow the veins. Other
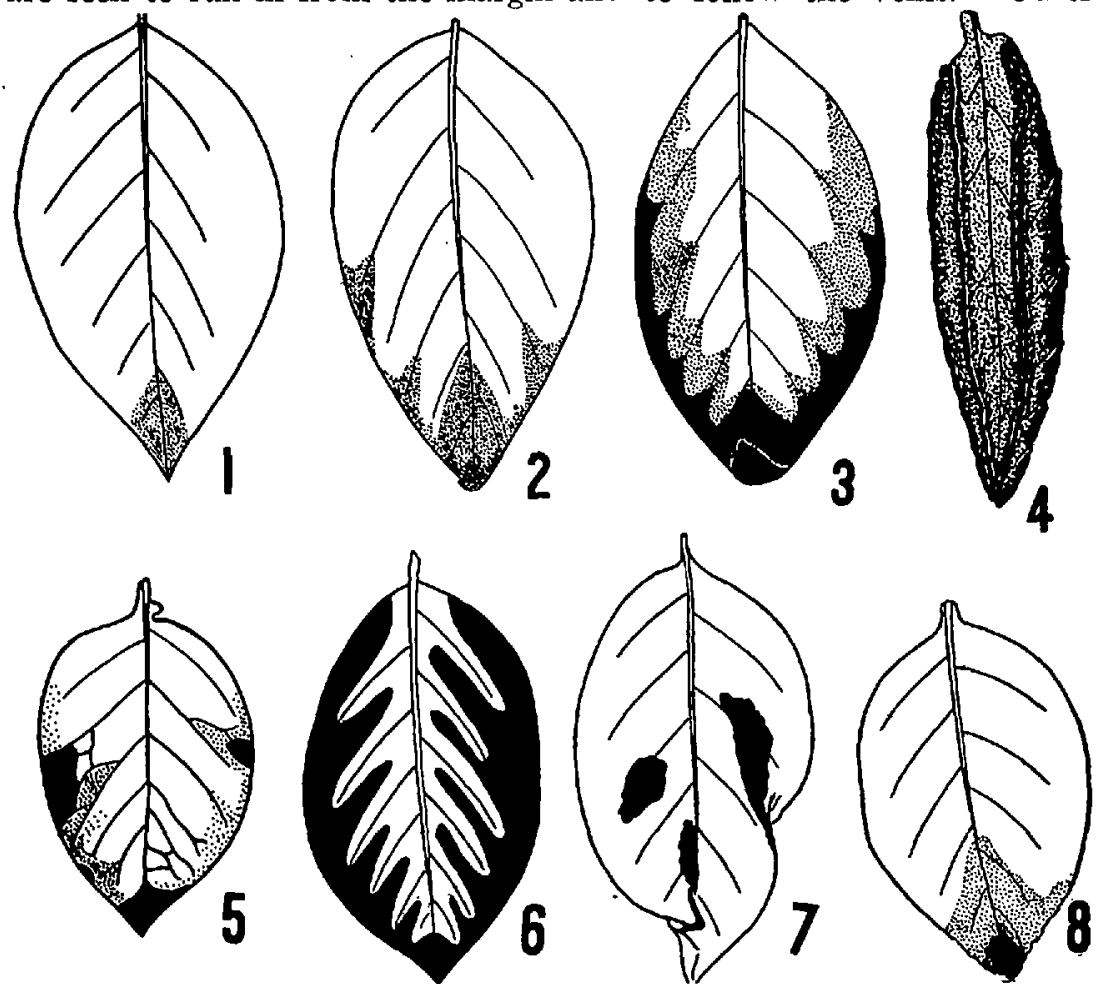

Fig. 15-Comparison of hopperburn with other leaf burns. 1-4. Successive stages of hopperburn; 5. tipburn (after Lutman); 6. scorching; 7 . sunscald; 8 . greenhouse burning. 
characteristics are the deep brown color of the burned areas, the curling upward and rolling in of the leaf margin and the crisp, fragile nature of the dead tissue. Tipburn as characterized by Lutman (Fig. 15, 5) has no relation to the venation, a fact which distinguishes it at once from hopperburn. The writers are not familiar with this type of burning which apparently is not common in Iowa. Burn injury(Fig. 15, 6) is rarely seen in the field but can be produced experimentally by placing leaves near intense heat. The burned areas in this case always run between the larger veins, the tissue immediately surrounding these being the last to die. Sun-scald (Fig.15, 7) is fairly common in Iowa but is not serious. It is noticed usually before the first signs of hopperburn appear and is the result of the action of the sun's rays through drops of water resting on leaves after a rain. These drops of water, if they are not evaporated or blown from the leaf soon, act as minute burning glasses and the tissue beneath burns brown. This injury is not correlated with veins or margins. The leaf is also distorted in the affected region by being folded. Another type of injury (Fig. 15, 8) is commonly seen on potato leaves in greenhouses. It resembles hopperburn in that it is correlated with the veins and begins at the margin and it also often appears on the lower and older leaves of the plant. It is distinct from hopperburn, however, in that the tissue only turns a comparatively light brown, there is little or no curling upward and rolling in of the leaf. and the burned area is more or less leathery in texture. Leaves affected with this disease appear limp and wilted. This is discussed in more detail in the following paragraphs.

\section{Effect of Mutilation of the Potato Leaves}

Preliminary experiments on the effect of mutilation of potato leaves have already been mentioned. ${ }^{1}$ This year these tests were repeated, using sterilized as well as non-sterilized instruments. The first series of tests consisted in pricking the larger veins with minuten Nadeln, once or several times both before and after these were sterilized. Seven days later the first signs of burning were noticed on several of the leaves so treated. This appeared in the form of a very small triangular brown area at the tip of the leaf and developed regardless of whether a sterilized or non-sterilized needle had been used. On the fifteenth day after the veins had been pierced all of the leaves but one showed this same type of injury.

Other leaves were similarly treated, using instead of the needles the fine, sharp ovipositors of two species of parasitic Hymenoptera.

'Fenton, F. A. Journal of Economic Entomology, Vol. 14, No. 1, pages 71-72, 1921. 
Of the two types of ovipositors used, one, the larger of the two, was comparatively short and stout, while the other was longer, finer and more flexible. These were used both before and after being sterilized. After 15 days a small burned area appeared at the tips of some of the leaves but several remained normal.

Finally, several leaves were mutilated by severing one or more of the larger veins and the midrib with a scalpel both before and after sterilizing the instrument. Seven days after this test a small dead area was noticed on one of the leaves mutilated in this manner, but this did not increase noticeably after this date.

\section{Discussion of Mutilaton Experiments}

In the above experiments it was noticed that the first signs of burning appeared suddenly on different plants on the same day. Possibly some abnormal change in temperature or humidity occurred on this date which was the real cause of the sudden appearance of burning. The fact that some check leaves showed this type of injury seems to substantiate this conclusion. Furthermore, the burned areas on the mutilated leaves were very small, and not at all similar to hopperburn. They did not enlarge in size nor were they preceded by any yellowing of the tissue, loss of turgidity, or curling upwards of the leaf as always accompanies typical hopperburn. The writers have repeatedly observed similar small brown areas on leaves that were not mutilated in any way and believe that under the abnormal conditions which are present in the greenhouse, the older leaves on a plant are very apt to show these areas. They feel therefore that they have not succeeded in producing anything which is at all comparable to hopperburn by mutilation of the leaves and that it is certainly not produced by the mere mechanical injury following feeding or oviposition by the leafhopper.

\section{Effect of Dilute Poisons on Potato Leaves}

Potato leaves were injected with five per cent hydrochloric acid and others with five per cent ammonium hydroxide by means of a hypodermic needle. Within 24 hours these leaves were wilted and shrivelled but there was no sign of burning. After this the leaves dried and the dead areas gradually turned a light brown color but the injury was in no way similar to hopperburn. Check leaves inoculated with water blanks remained normal.

\section{Inoctlation of Hopperburn Tissue Solution into Potato Leaves}

On July 18 a small amount of solution prepared from a macerated potato leaf showing a trace of hopperburn was injected 
into normal potato leaves. Other leaves were injected likewise with a solution prepared from a leaf showing advanced stages of this disease. Finally, leaves were inoculated with a solution prepared from a leaf that had been killed by hopperburn. By July 25 some of the leaves showed a slight browning at the tip and on July 30 all showed this same type of injury. On August 10 this experiment was closed as the injured area on the inoculated leaves had not increased and they were clearly not affected with hopperburn. The injury in this case was identical with that resulting from multilation and was probably a result of greenhouse conditions.

Inoculation of Potato Leaves with E. mali Extract by Means of a Hypodermic Needle

On July 19 approximately 100 Empoasca mali adults of both sexes were collected and macerated in $10 \mathrm{cc}$. of water that had been previously distilled and sterilized. Three potato leaflets were inoculated with the liquid drawn off from this solution and also the petiole of a single leaf was inoculated in the same manner. On July 25 there was a scar or lesion noticed in the midvein of each leaf where the needle had been pricked in. At the same time two of the leaves showed decided burning similar in every way to hopperburn, while the third showed the burning less distinctly. The injury increased daily and on July 30 there was no question but that a disease very similar to hopperburn had been produced in every case. On July 29 the above experiment was repeated, using in these inoculations a solution made from 58 adults of both sexes. Part of the leaves in this test were reinoculated 24 hours later by a freshly prepared solution made from 56 adults of both sexes. In this latter test a slight burning was produced but it was in no way comparable to the injury obtained by the first set of inoculations. Although fewer insects were used this was more than compensated for by the additional inoculation the following day.

On July 19 approximately 88 nymphs mostly in the fourth and fifth instars were macerated in $10 \mathrm{cc}$. of water that had been previously distilled and sterilized. Four inoculations were made of which three were in leaf tissue and one in a leaf petiole. Only one of the leaves so treated showed any signs of hopperburn injury, and on this one there was a small brown area at the tip. In all cases, however, there was a marked lesion produced at the point of inoculation. Here the tissue seemed to collapse and the mid-vein became distorted. In dried specimens these lesions are very distinct, showing that the nymphs 
must have contained a toxic principle. Inoculation of check leaves with water blanks failed to produce injury.

\section{Inoculation of Potato Leaves by Needle Punctures}

On July 27, 80 nymphs in advanced stages were macerated in a small quantity of water. Small drops of this solution were placed on the under surface of the leaves and then using a very fine needle, the leaves were punctured 25 times. This procedure was repeated July 28,29 and 30. July 31 one leaf so treated showed a slight burning at the tip but the others were normal. August 2 all leaves so treated but one showed a slight curling. August 10 this experiment was closed, there being no sign of hopperburn on any of the leaves, although there was a large scar at the point of inoculation.

Inoculation by Means of Crushing the Nymph on a Leaf Abrasion

Having failed to get positive results in the above tests, it was decided to crush the live insects on the leaf, making in so doing a cut in the leaf surface. Three fifth instar nymphs were crushed near a large side vein of one leaf; one each day, on July 27,28 and 29, respectively. At each point of inoculation the tissue turned yellow and then brown, but the injury was very localized and the rest of the leaf remained perfectly normal. A second leaf received the same treatment except that on the first day a fourth instar nymph was used and the results were the same. Thirteen nymphs in various stages were crushed on a third leaf during a three day period and in this experiment the injury was much more noticeable. Not only did the tissue immediately beneath the crushed nymph turn brown but also the area surrounding it was affected in the same way. This was proof that the nymphs contain a toxic substance but in small quantities.

\section{Inoculation of Potato Leaves by Leafhopper Residue}

On July 29 the residue left over from 58 crushed adults in the needle inoculation experiments mentioned above was placed on potato leaves and leaf petioles and pricked in with a fine scalpel. Twenty-four hours later the tissue beneath had whitened and there was a decided lesion. However, the injury continued to remain localized and the leaves were apparently little affected by this except in one case. Here the lesion on the petiole was so pronounced that it was nearly girdled. This experiment demonstrated that the adults apparently possess the same toxic substance as the nymphs and further it partially explained the lack of better results in other inoculation tests; namely, that the mere 
crushing of the insects apparently fails to dissolve enough of this toxic substance to produce injury in all cases.

\section{Experiments with Potometer}

It was thought that it might be possible to get more of the leafhooper solution into the leaves by cutting them off and placing the stems in the solution so that it would be drawn up into the leaf by transpiration. Accordingly, a number of hollow glass tubes were bent into a " $U$ " shape, a rubber tube was placed securely over one end of the glass tube and then into this rubber tube the stem of an entire leaf was firmly placed and held there by grafting wax. After trying this out and finding that most of the leaves remained fresh for some time under these conditions with water in the tube, a solution made from 50 crushed nymphs was poured into one of the tubes. The diameter of the tube was so small that it was possible to fill it with a highly concentrated extract. As this was darker colored than the water it could be easily seen that the extract was being drawn up into the leaf which promptly became flaccid and remained so without entirely wilting for five days. At the end of five days, on August 29, the leaf was removed. It had yellowed in certain areas, and there had been a tendency for the margin to roll up, a condition similar to the beginning of hopperburn. It was found that the cut end of the stem had partially decayed, a condition brought about by the fermentation of the insect remains in the water. The decayed part was cut off and the leaf placed with the stem in water. It soon freshened up and August 31 the leaf had recovered except in the injured portion. The injury resembled hopperburn very closely. Leaves in plain water remained perfectly fresh and showed no injury throughout this test, provided the apparatus was working properly. This experiment was repeated later with the difference that only the clear solution free from the insect remains was used, thus avoiding decay of the leaf due to fermentation. The leaf became flaccid as in the other case and then developed symptoms of hopperburn.

\section{Discussion of Inoculation Results}

The writers were at a loss at first to understand the variable resuits in the different inoculation tests with hypodermic needles as described in the preceding paragraphs. It was noted, for example, that extracts from crushed adults caused marked symptoms of hopperburn in one series of inoculations and negative results in another. Furthermore, it was noted that extract from crushed nymphs produced less abnormalities in the leaf tissue than from the macerated adults. This would indicate that 
the nymphs contained less of the toxic substance than the adults, a fact not born out by previous experiments', where it was shown that the nymph is the more toxic of the two. Undoubtedly some difference in procedure not noted at the time may have been the cause of this. Failure to produce burning in all cases may have been due to inability to force enough of the liquid into the plant by means of the hypodermic needle. This represented a real difficulty in our earlier inoculation tests as the natural turgidity of the leaf made it almost impossible to force much of the extract in. The difference in the amount of extract injected into the leaves as a result of this turgidity may explain the difference in the effects of the toxin in the leaf. That the negative results of the earlier experiments may have been due to the failure to introduce enough of the hopper extract to produce burning because of the natural turgidity of the leaves is also indicated by the success of the tests with a potometer. Another factor entering in was the difficulty of getting very much of the toxic substance into solution by a mere maceration of the insects in distilled water. Apparently not all or perhaps not even much of the toxic substance was extracted by this method as was indicated by the highly toxic action on potato leaves, of the residue composed of macerated insects after the clear solution had been drawn off. That the live nymphs do contain this toxic substance was demonstrated a number of times by crushing them over a leaf abrasion. Very distinct lesions as already described resulted in every case.

The above tests need to be repeated on a larger scale and some better method devised for getting the toxin into the solution. Furthermore, extracts prepared from other insect species should be injected into the leaves as it is well known that insect sera often contain toxic principles.

\section{EFFECT OF BORDEAUX MIXTURE ON EMPOASCA MALI}

By F. A. Fenton and Albert Hartzell, Iowa Siate College, Ames, Iowa

Since the establishment of the fact that Empoasca mali LeB. is the cause of potato tipburn, it was found that Bordeaux mixture in some way prevented this foliage disease by its action on this insect. It was thought at first that it acted as a repellent ${ }^{2}$ because plants sprayed with this fungicide were noticed to have comparatively few leafhoppers on them. The senior author ${ }^{3}$ demonstrated later that this com-

1Fenton, F. A. loc. cit. pages 76-77.

3Fluke, C. L. Journal of Economic Entomology, Vol. 12, 1919, pages 256-257.

'Fenton, F. A. Journal of Economic Entomology, Vol. 14, 1921, pages 71-79. 